\title{
Intersection cohomology of representation spaces of surface groups
}

\author{
Young-Hoon Kiem \\ Department of Mathematics \\ Stanford University \\ Stanford, CA 94305 \\ kiem@math.yale.edu
}

\section{Introduction}

Let $\Sigma$ be a closed Riemann surface of genus $g \geq 2$ and $\pi$ be its fundamental group. The representation space

$$
X(G)=\operatorname{Hom}(\pi, G) / G
$$

of $\pi$ into a compact connected Lie group $G$ has been playing important roles in differential geometry (the moduli space of flat connections), algebraic geometry (the moduli space of holomorphic principal bundles) and topology (Casson's invariant). This is a compact pseudomanifold which can be thought of as the symplectic reduction of a Hamiltonian $G$-space, the extended moduli space. The purpose of this paper is to study the local structure of $X(G)$ in terms of the symplectic geometry of the extended moduli space and to show by the splitting theorem [Kie, KW] that its intersection cohomology is embedded into the $G$-equivariant cohomology of $\operatorname{Hom}(\pi, G)$, which can be computed by the equivariant Morse theory of Atiyah-Bott AB82 $\S 10$ at least in principle. The image is identified with a subspace $V_{U}^{*}$ truncated locally and this enables us to compute the intersection cohomology as a graded vector space with a nondegenerate pairing.

The extended moduli space $\mathcal{N}^{\mathfrak{g}}$ for a simply connected compact Lie group $G$ was defined and studied by L. Jeffrey Jef94. Roughly, it is a "partial reduction" of the space of connections by the based gauge group and thus 
carries a symplectic structure on the smooth part together with a (residual) $G$ action. $X(G)$ is now the symplectic reduction of this partial reduction. In JK598, L. Jeffrey and F. Kirwan applied their nonabelian localization principle to the extended moduli space and proved Witten's formulas for the intersection numbers of the moduli spaces of vector bundles of coprime rank and degree. In $\S 2$, we review the definition and some key results for $\mathcal{N}^{\mathfrak{g}}$. Then we determine the symplectic slice at a point in the zero set of the moment map. As is well-known, at a smooth point in $X(G)$, the tangent space is isomorphic to the symplectic slice. Our description of the symplectic slice is consistent with W. Goldman's description of the tangent space [Gol84].

Given a singular reduction, R. Sjamaar and E. Lerman provided a useful local description [SL91] and showed that a singular reduction is a stratified space, symplectic on each stratum. In $\S 3$, we apply their results to study the local structures of $X(G)$. Locally, $X(G)$ looks like the symplectic reduction of the symplectic slice by a linear action of the stabilizer. This gives us a local description of $X(G)$, consistent with the result of Goldman-Millson in GM88, where they obtained analytic results through deformation theory.

Since $X(G)$ is a singular pseudomanifold, intersection cohomology is an interesting invariant, at least as important as the ordinary cohomology because of its nice properties including Poincaré duality in spite of singularities. Generalizing the results of [Kie], we showed in [KW] that the intersection cohomology of a reduced space $M / / G$ of a Hamiltonian $G$ space $M$ with proper moment map $\mu$ is naturally isomorphic to a subspace, called the truncated equivariant cohomology, of the equivariant cohomology $H_{G}^{*}\left(\mu^{-1}(0)\right)$ under an assumption named weakly balanced. This condition was devised to make the codimensions of the unstable strata in the blow-ups in the partial desingularization process, large enough. The truncated equivariant cohomology is defined by truncating the equivariant cohomology along each stratum and the splitting theorem 1 enables us to compute the Betti numbers and intersection pairing of $I H^{*}(M / / G)$ in terms of the equivariant cohomology. In $\S 4$, we recall this theorem and show that the extended moduli space satisfies the assumption. Therefore, we can compute the intersection cohomology of $X(G)$ in terms of the $G$ equivariant cohomology of $\operatorname{Hom}(\pi, G)$.

The case where $G=S U(2)$ is discussed in $\S 5$. We can compute the intersection cohomology groups of $X(S U(2))$ by using the structure theorem of $H_{S U(2)}^{*}(\operatorname{Hom}(\pi, S U(2)))$ from [Kie00].

\footnotetext{
${ }^{1}$ The isomorphism is a splitting of the Kirwan map $\kappa: H_{G}^{*}\left(\mu^{-1}(0)\right) \rightarrow I H^{*}\left(\mu^{-1}(0) / G\right)$.
} 
If the Lie group $G$ is not simply connected, $X(G)$ is not connected. In fact, $\pi_{0}(X(G)) \cong \pi_{1}(G)$. Let $\tilde{G}$ denote the universal cover of $G$ with (finite) fiber $C$. Then there is a covering space $X(\tilde{G}) \rightarrow X(G)$ onto a component we denote by $X(G)_{0}$. Then the intersection cohomology of $X(G)_{0}$ is the $C^{2 g}$ invariant part of the intersection cohomology of $X(\tilde{G})$ which can be computed by the splitting theorem.

Every cohomology group in this paper has complex coefficients and every intersection cohomology has middle perversity.

Acknowledgements. I am grateful to Professors Ronnie Lee, Lisa Jeffrey, Frances Kirwan and Jon Woolf for useful discussions.

\section{Extended moduli space}

Let $G$ be a simply connected compact Lie group and $\Sigma$ be a closed Riemann surface of genus $g \geq 2$. Let $\pi$ denote the fundamental group of $\Sigma$. The representation space

$$
X(G):=\operatorname{Hom}(\pi, G) / G
$$

can be realized as the symplectic reduction of a Hamiltonian $G$ space $\mathcal{N}^{\mathfrak{g}}$, called the extended moduli space. This was defined and studied by L. Jeffrey in [Jef94.

Definition 2.1 Let $\mathfrak{g}=\operatorname{Lie}(G)$. We define

$$
\mathcal{N}^{\mathfrak{g}}:=\left\{\left(a_{1}, \cdots, a_{2 g}, \Lambda\right) \in G^{2 g} \times \mathfrak{g} \mid \prod\left[a_{i}, a_{i+g}\right]=e^{\Lambda}\right\}
$$

More generally, for each central element c in $G$, we define

$$
\mathcal{N}_{c}^{\mathfrak{g}}:=\left\{\left(a_{1}, \cdots, a_{2 g}, \Lambda\right) \in G^{2 g} \times \mathfrak{g} \mid \prod\left[a_{i}, a_{i+g}\right]=c e^{\Lambda}\right\} .
$$

Let $P: G^{2 g} \times \mathfrak{g} \rightarrow G$ be the map defined by

$$
P\left(a_{1}, \cdots, a_{2 g}, \Lambda\right)=\prod\left[a_{i}, a_{i+g}\right] e^{-\Lambda} .
$$

Then $\mathcal{N}^{\mathfrak{g}}=P^{-1}(1) . d P_{(a, \Lambda)}$ is surjective if $P(a, \Lambda)=1$ and $\Lambda=0$ because $d(\exp )_{0}$ is surjective. Hence, $\mathcal{N}^{\mathfrak{g}}$ is smooth in a neighborhood of

$$
\operatorname{Hom}(\pi, G)=\left\{\left(a_{1}, \cdots, a_{2 g}\right) \in G^{2 g} \mid \prod\left[a_{i}, a_{i+g}\right]=1\right\}
$$


It is not hard to see that $\mathcal{N}^{\mathfrak{g}}$ is connected. Also, from the work of A. Ramanathan Ram75] and Atiyah-Bott [AB82, it is easy to see that $\operatorname{Hom}(\pi, G)$ is connected.

The extended moduli space can be considered as a moduli space of flat connections as follows. Delete an open disk $D$ with boundary $S$ from $\Sigma$ and denote the resulting surface by $\Sigma^{\prime}$. Fix a parametrization of $S$ by a parameter $s \in \mathbb{R} / \mathbb{Z}$ and a diffeomorphism of a neighborhood of $S$ to $\mathbb{R} / \mathbb{Z} \times[0, \epsilon)$. Let $\mathcal{A}_{\text {flat }}^{\mathfrak{g}}\left(\Sigma^{\prime}\right)$ denote the set of flat connections on $\Sigma^{\prime}$ whose restriction to a neighborhood of $S$ is $\alpha d s$ for some $\alpha \in \mathfrak{g}$. Let $\mathcal{G}_{0}\left(\Sigma^{\prime}\right)$ be the space of maps $g: \Sigma^{\prime} \rightarrow G$ such that $g=I \in G$ in a neighborhood of $S$. Then, as usual, holonomy defines a map $\mathcal{A}_{\text {flat }}^{\mathfrak{g}}\left(\Sigma^{\prime}\right) / \mathcal{G}_{0}\left(\Sigma^{\prime}\right) \rightarrow \mathcal{N}^{\mathfrak{g}}$. We quote the following theorem from [Jef94.

Theorem 2.2 $\mathcal{N}^{\mathfrak{g}}$ is homeomorphic to $\mathcal{A}_{\text {flat }}^{\mathfrak{g}}\left(\Sigma^{\prime}\right) / \mathcal{G}_{0}\left(\Sigma^{\prime}\right)$.

From this gauge theoretic description, we see that the tangent space at a smooth point in $\mathcal{N}^{\mathfrak{g}}$ is isomorphic to the first cohomology $\tilde{H}^{1}$ of the complex (see [Def94])

$$
\Omega_{c}^{0}\left(\Sigma^{\prime}\right) \otimes \mathfrak{g} \stackrel{d_{A}}{\longrightarrow} \Omega^{1, \mathfrak{g}}\left(\Sigma^{\prime}\right) \longrightarrow \Omega_{c}^{2}\left(\Sigma^{\prime}\right) \otimes \mathfrak{g}
$$

where $\Omega_{c}^{i}$ is the space of $i$-forms with compact support in the interior of $\Sigma^{\prime}$ and $\Omega^{1, \mathfrak{g}}\left(\Sigma^{\prime}\right)=\left\{b \in \Omega^{1}\left(\Sigma^{\prime}\right) \otimes \mathfrak{g} \mid b=\alpha d s\right.$ in a neighborhood of $S$ for some $\left.\alpha \in \mathfrak{g}\right\}$. Notice that $\Omega^{1, \mathfrak{g}}\left(\Sigma^{\prime}\right) \cong \Omega_{c}^{1}\left(\Sigma^{\prime}\right) \otimes \mathfrak{g} \oplus \mathfrak{g}$ by choosing a 1 -form whose restriction to a neighborhood of $S$ is $d s$. Hence, the index of the complex is $-2 g(\operatorname{dim} G)$ since the Euler characteristic of $\Sigma^{\prime}$ is $1-2 g$. Therefore, the dimension of $\mathcal{N}^{\mathfrak{g}}$ is $2 g(\operatorname{dim} G)$.

Let $a, b \in \Omega^{1, \mathfrak{g}}\left(\Sigma^{\prime}\right)$ and define a pairing $\omega$ on $\Omega^{1, \mathfrak{g}}\left(\Sigma^{\prime}\right)$ by

$$
\omega(a, b)=\int_{\Sigma^{\prime}}\langle a, b\rangle
$$

where $\langle$,$\rangle is the nondegenerate invariant metric on \mathfrak{g}$. By Stokes' theorem, $\omega$ defines a 2 -form on $\tilde{H}^{1}$, which is shown to be symplectic in [Jef94]. Thus near $\operatorname{Hom}(\pi, G), \mathcal{N}^{\mathfrak{g}}$ is a symplectic manifold.

For $\left(a_{1}, \cdots, a_{2 g}, \Lambda\right) \in \mathcal{N}^{\mathfrak{g}}$ and $h \in G$, consider the action

$$
h \cdot\left(a_{1}, \cdots, a_{2 g}, \Lambda\right)=\left(h a_{1} h^{-1}, \cdots, h a_{2 g} h^{-1}, h \Lambda h^{-1}\right) .
$$

This makes $\mathcal{N}^{\mathfrak{g}}$ a $G$ space. In terms of gauge theory, this action can be described by the corresponding action on the space of connections. Suppose 
$\zeta \in \mathfrak{g}$ and let $a \in \Omega^{1, \mathfrak{g}}\left(\Sigma^{\prime}\right)$ be a $d_{A^{-}}$closed 1 -form whose restriction to a neighborhood of $S$ is $\alpha d s$. Then

$$
\omega\left(d_{A} \zeta, a\right)=\int_{\Sigma^{\prime}}\left\langle d_{A} \zeta, a\right\rangle=\langle\zeta, \alpha\rangle .
$$

Let $\mu: \mathcal{N}^{\mathfrak{g}} \rightarrow \mathfrak{g}$ be the map defined by $\mu\left(a_{1}, \cdots, a_{2 g}, \Lambda\right)=-\Lambda$. Then the above computation shows that $\mu$ is a moment map for the $G$ action on a neighborhood $U=\mu^{-1}(B)$, for a small open ball $B$ around 0 , of $\operatorname{Hom}(\pi, G)=\mu^{-1}(0)$. In particular, the representation space $X(G)$ is the symplectic reduction $U / / G$ of $U$ by the $G$ action.

Near a point $\phi=[A]$ in $\mu^{-1}(0), \mathcal{N}^{\mathfrak{g}}$ is diffeomorphic to $\tilde{H}^{1}$ of (11). The orbit direction is given by $d_{A} \zeta$ for $\zeta \in \mathfrak{g}$ and thus by (3) its symplectic orthogonal complement in $\tilde{H}^{1}$ is precisely $H_{c}^{1}\left(\Sigma^{\prime} ; d_{A}\right) \cong H^{1}\left(\Sigma, D ; d_{A}\right)$. Notice that the local system extends to $\Sigma$ because $\phi=[A] \in \mu^{-1}(0)$. The infinitesimal action of $\mathfrak{g}$ is given by the image of the map

$$
H^{0}\left(D ; d_{A}\right) \rightarrow H^{1}\left(\Sigma, D ; d_{A}\right)
$$

Therefore, from the long exact sequence, we deduce the following theorem.

Theorem 2.3 The symplectic slice at a point $\phi=[A]$ in $\mu^{-1}(0) \subset \mathcal{N}^{\mathfrak{g}}$ is $H^{1}\left(\Sigma ; d_{A}\right)=H^{1}\left(\pi ; \mathfrak{g}_{A d \phi}\right)$.

Hence, the tangent space at a smooth point $\phi$ of $X(G)$ is isomorphic to $H^{1}\left(\pi ; \mathfrak{g}_{A d \phi}\right)$. This is consistent with Goldman's result [Gol84. From (2), the symplectic structure on the slice is given by the cup product and $\langle$,$\rangle .$

\section{Local description}

In this section, we apply the results of Sjamaar-Lerman [SL91] to study the local structure of $X(G)$ and $U \subset \mathcal{N}^{\mathfrak{g}}$ with the $G$ action.

Let $M$ be a Hamiltonian $G$ space with moment map $\mu$. We recall the following local normal form theorem.

Theorem 3.1 SL91 Let $p \in \mu^{-1}(0)$ and $\hat{W}_{p}$ be the symplectic slice of the orbit Gp. Then a neighborhood of the orbit is equivariantly symplectomorphic to a neighborhood of the zero section of

$$
G \times \times_{\text {Stabp }}\left((\mathfrak{g} / \mathfrak{h})^{*} \times \hat{W}_{p}\right)
$$

\footnotetext{
${ }^{2}$ The sign depends on the convention.
} 
with moment map $\mu(a, \xi, v)=A d^{*}(a)\left(\xi+\mu_{\hat{W}}(v)\right)$ where $\mu_{\hat{W}}: \hat{W}_{p} \rightarrow \mathfrak{h}^{*}$ is the moment map for the linear Stabp action and $\mathfrak{h}=\operatorname{Lie}(\operatorname{Stab} p)$.

As a consequence, a neighborhood of $x \in \mu^{-1}(0) / G$, corresponding to $p$, is homeomorphic to $\hat{W}_{p} / / S t a b p$ which is the reduction of $G \times{ }_{S t a b p}\left((\mathfrak{g} / \mathfrak{h})^{*} \times \hat{W}_{p}\right)$ by $G$.

In the case of extended moduli space, we showed in the previous section that the symplectic slice at a point $\phi \in \operatorname{Hom}(\pi, G)$ is $\hat{W}_{\phi}=H^{1}\left(\pi, \mathfrak{g}_{A d \phi}\right)$ on which Stabp acts by conjugation on $\mathfrak{g}$. The moment map for this action is given by $\mu_{\hat{W}_{\phi}}(u)=[u, u], \mu_{\hat{W}_{\phi}}: H^{1}\left(\pi, \mathfrak{g}_{A d \phi}\right) \rightarrow H^{2}\left(\pi, \mathfrak{g}_{A d \phi}\right) \cong \mathfrak{h}^{*}$ using the Lie bracket of $\mathfrak{g}$ and the cup product.

The above local normal form theorem now shows that in a neighborhood of the image of a point $\phi \in \operatorname{Hom}(\pi, G), X(G)$ is homeomorphic to the symplectic reduction $H^{1}\left(\pi, \mathfrak{g}_{A d \phi}\right) / / S t a b \phi$. This is again consistent with the deformation theory result of Goldman-Millson GM88 but our approach is more in the spirit of symplectic geometry.

Let $\mathfrak{h}=\operatorname{Lie}(\operatorname{Stab} \phi)$ and $\mathfrak{z}(\mathfrak{h})=\left\{\zeta \mid\left[\zeta, \zeta^{\prime}\right]=0\right.$ for all $\left.\zeta^{\prime} \in \mathfrak{h}\right\}$. Recall that $H^{1}\left(\pi, \mathfrak{g}_{A d \phi}\right)$ is the quotient of the space of cocycles

$$
Z^{1}\left(\pi, \mathfrak{g}_{A d \phi}\right)=\{u: \pi \rightarrow \mathfrak{g} \mid u(r s)=u(r)+A d \phi(r) u(s)\}
$$

by the space of coboundaries

$$
B^{1}\left(\pi, \mathfrak{g}_{A d \phi}\right)=\left\{u: \pi \rightarrow \mathfrak{g} \mid u(r)=A d \phi(r) u_{0}-u_{0} \text { for } u_{0} \in \mathfrak{g}\right\} .
$$

Since $A d \phi$ stabilizes $\mathfrak{h}, A d \phi$ preserves $\mathfrak{z}(\mathfrak{h})$ and $\mathfrak{z}(\mathfrak{h})^{\perp}$ for the orthogonal decomposition $\mathfrak{g}=\mathfrak{z}(\mathfrak{h}) \oplus \mathfrak{z}(\mathfrak{h})^{\perp}$. Hence, each $u: \pi \rightarrow \mathfrak{g}$ is the sum of cocycles $u_{\mathfrak{h}}: \pi \rightarrow \mathfrak{g} \rightarrow \mathfrak{z}(\mathfrak{h})$ and $u_{\mathfrak{h}}^{\perp}: \pi \rightarrow \mathfrak{g} \rightarrow \mathfrak{z}(\mathfrak{h})^{\perp}$. Thus, we get the induced decomposition

$$
H^{1}\left(\pi, \mathfrak{g}_{A d \phi}\right)=H^{1}\left(\pi, \mathfrak{z}(\mathfrak{h})_{A d \phi}\right) \oplus H^{1}(\pi, \mathfrak{z}(\mathfrak{h}) \stackrel{\perp}{A d \phi}) .
$$

In terms of gauge theory, this is obtained by reduction of the structure group to the centralizer $H^{\prime}$ of the identity component $H$ of $S t a b(\phi)$. Clearly, the first factor is $\mathfrak{h}$ fixed while the second factor has no nonzero fixed point. [3 Hence, the $\mathfrak{h}$-fixed subspace in $\hat{W}_{\phi}$ is $\hat{W}_{\phi}^{\mathfrak{h}}:=H^{1}\left(\pi, \mathfrak{z}(\mathfrak{h})_{\text {Add }}\right)$ and its orthogonal complement is $W_{\phi}:=H^{1}(\pi, \mathfrak{z}(\mathfrak{h}) \stackrel{\perp}{A} d \phi)$

\footnotetext{
${ }^{3}$ One easy way to see it is to consider $H^{1}\left(\pi, \mathfrak{z}(\mathfrak{h}) \frac{\perp}{A} d \phi\right)$ as the space of harmonic $\mathfrak{z}(\mathfrak{h})^{\perp}$ valued 1-forms.
} 
In fact, the (orbifold) stratum direction $\hat{W}_{\phi}^{\mathfrak{h}}$ can be integrated: since $\mathfrak{h}=\mathfrak{z}(\phi)$ and $\mathfrak{z}(\mathfrak{z}(\mathfrak{h}))=\mathfrak{h}, \phi$ is an irreducible point in $\operatorname{Hom}\left(\pi, H^{\prime}\right)$ where $H^{\prime}$ is the centralizer of $H=\operatorname{Stab}(\phi)$. We get the following decompositions

$$
\begin{aligned}
\operatorname{Hom}(\pi, G) & =\cup_{(H) \in I} G \operatorname{Hom}\left(\pi, H^{\prime}\right)^{i r r} \\
\operatorname{Hom}(\pi, G) / G & =\cup_{(H) \in I} \operatorname{Hom}\left(\pi, H^{\prime}\right)^{i r r} / N^{H^{\prime}}
\end{aligned}
$$

whose $\mathrm{irr}$ denotes the irreducible part.

\section{Weakly balanced action and the splitting theorem}

In this section, we show that the $G$ action on the extended moduli space is weakly balanced in the sense of $\mathrm{Kie}, \mathrm{KW}$ and hence we get an isomorphism of the truncated equivariant cohomology of $\operatorname{Hom}(\pi, G)$ with the intersection cohomology of the representation space $X(G)$.

First, we recall the weakly balanced condition. Let $U$ be a Hamiltonian $G$ space with moment map $\mu$ and $Z=\mu^{-1}(0)$. Let $H$ be the identity component of $S t a b p$ for a point $p \in Z$ and $\hat{W}_{p}$ denote the symplectic slice at $p$. We decompose $\hat{W}_{p}=\hat{W}_{p}^{\mathfrak{h}} \oplus W_{p}$ into the $H$ fixed subspace and its complement. Choose a compatible complex structure on the symplectic vector space $W_{p}$ and let $\mathbb{P} W_{p}$ denote the complex projective space associated to $W_{p}$. The unstable strata in $\mathbb{P} W_{p}$ are parametrized by a finite set $\mathcal{B}$ in the Weyl chamber. Namely, $\mathcal{B}$ is the set of the closest points from the origin to the convex hulls of some weights of the action of $H$ on $W_{p}$. (See Kir84.) Given $\beta \in \mathcal{B}$, let $n(\beta)$ denote the number of weights $\alpha$ such that $\langle\alpha, \beta\rangle<\langle\beta, \beta\rangle$ with respect to the invariant metric.

Definition 4.1 We say the $H$ action on $W_{p}$ is linearly balanced if $2 n(\beta) \geq$ $\frac{1}{2} \operatorname{dim} W_{p}$.

This condition makes the codimensions of the unstable strata large enough.

For any subgroup $K \subset H$ which also appears as the identity component of the stabilizer of a point in $Z$, we consider the $N^{K} \cap H / K$ action on the $K$ fixed point subspace $W_{p}^{\mathfrak{k}}$ where $N^{K}$ is the normalizer of $K$.

\footnotetext{
${ }^{4}$ Here, "irreducible" means that the infinitesimal stabilizer is of minimal dimension.
} 
Definition 4.2 We say the $G$ action on a Hamiltonian space $U$ is balanced if for all $p \in Z$ the action of the identity component $H$ of its stabilizer on $W_{p}$ is linearly balanced and if for a subgroup $K$ as above the $N^{K} \cap H / K$ action on $W_{p}^{\mathfrak{k}}$ is also linearly balanced.

The usefulness of this condition becomes evident from the splitting theorem in [Ki], [KW]. To describe this theorem, we need to recall the "truncated equivariant cohomology". For each connected subgroup $H$ of $G$, which is the identity component of the stabilizer of a point in $Z$, let $Y_{H}$ denote the subset of $Z$ fixed by $H$. Consider the obvious map

$$
G Y_{H} \leftarrow G \times_{N^{H}} Y_{H}
$$

This composed with the inclusion induces a map of equivariant cohomology groups

$$
H_{G}^{*}(Z) \rightarrow H_{G}^{*}\left(G Y_{H}\right) \rightarrow H_{N^{H}}^{*}\left(Y_{H}\right)
$$

which we denote by $\Psi_{H}$. Note that $H_{N^{H}}^{*}\left(Y_{H}\right) \cong\left[H_{N_{0}^{H} / H}^{*}\left(Y_{H}\right) \otimes H_{H}^{*}\right]^{\pi_{0} N^{H}}$ where $H_{H}^{*}$ denotes the cohomology of the classifying space of $H$ and $N_{0}^{H}$ is the identity component of $N^{H}$. We define the truncated equivariant cohomology by

$$
V_{U}^{*}:=\left\{\xi \in H_{G}^{*}(Z) \mid \Psi_{H}(\xi) \in H_{N_{0}^{H} / H}^{*}\left(Y_{H}\right) \otimes H_{H}^{<n_{H}}\right\}
$$

where $n_{H}=\frac{1}{2} \operatorname{dim}\left(W_{p} / / H\right)$ for $p \in Z$ whose stabilizer has $H$ as its identity component.

Theorem 4.3 Kie, KW] Suppose the proper Hamiltonian $G$ space $U$ is balanced. Then there is an isomorphism $\rho: I H^{*}(X) \rightarrow V_{U}^{*}$ such that the intersection pairing $\langle$,$\rangle of I H^{*}(X)$ is given by

$$
\rho(\alpha) \cup \rho(\beta)=\langle\alpha, \beta\rangle \rho(\tau)
$$

for all $\alpha, \beta \in I H^{*}(X)$ of complementary degrees with respect to the real dimension of $X=U / / G$ where $\tau$ is the fundamental class.

In fact, the theorem is proved in a more general context, namely under the "weakly balanced" assumption. For our purpose in this paper, we will need only the balanced condition.

Let us now return to the extended moduli space. For each point $\phi$ in $\mu^{-1}(0)=\operatorname{Hom}(\pi, G)$, we have $W_{\phi}=H^{1}\left(\pi, \mathfrak{z}(\mathfrak{h})_{A}^{\perp} d \phi\right)$ where Stab $\phi$ acts on 
$\mathfrak{z}(\mathfrak{h})^{\perp}$ by conjugation and $\mathfrak{h}=\operatorname{Lie}(\operatorname{Stab} \phi)$. There is a geometric way to assign a compatible complex structure on this space. Choose a complex structure on $\Sigma$ and consider the holomorphic vector bundle over $\Sigma$ with fiber $\mathfrak{z}(\mathfrak{h})^{\perp} \otimes \mathbb{C}$ given by $\phi$

$$
E=\tilde{\Sigma} \times_{\pi}\left(\mathfrak{z}(\mathfrak{h})^{\perp} \otimes \mathbb{C}\right) \rightarrow \Sigma
$$

where $\tilde{\Sigma}$ is the universal cover. Then $W_{\phi}$ is canonically isomorphic to the complex vector space $H^{0,1}\left(\Sigma, \mathfrak{z}(\mathfrak{h})^{\perp} \otimes \mathbb{C}\right)$.

Consider the root space decomposition of the complex semisimple Lie algebra $\mathfrak{g} \otimes \mathbb{C}$ with respect to a Cartan subalgebra of $\mathfrak{g}$ containing a Cartan subalgebra $\mathfrak{t}_{\mathfrak{h}}$ of $\mathfrak{h}$. Of course, $\lambda$ is a root iff $-\lambda$ is a root. Hence, the weights of the $\mathfrak{t}_{\mathfrak{h}}$ action on $\mathfrak{g}$ are symmetric with respect to the origin and clearly $\mathfrak{z}(\mathfrak{h}) \otimes \mathbb{C}$ lies in the zero eigenspace. Therefore, the weights of the $\mathfrak{t}_{\mathfrak{h}}$ action on $\mathfrak{z}(\mathfrak{h})^{\perp} \otimes \mathbb{C}$ are symmetric with respect to the origin. Now, because $\mathfrak{t}_{\mathfrak{h}}$ commutes with $\phi$, the eigenspace decomposition of the fiber $\mathfrak{z}(\mathfrak{h})^{\perp} \otimes \mathbb{C}=$ $\bigoplus_{\lambda} F_{\lambda}$ provides us with a decomposition $E=\bigoplus_{\lambda} E_{\lambda}$ of the vector bundle into subbundles of eigenspaces. The degrees of the $E_{\lambda}$ are all zero and by the invariant metric of $\mathfrak{g}, E_{\lambda} \cong E_{-\lambda}^{*}$. In particular, the subbundles $E_{\lambda}, E_{-\lambda}$ have the same Riemann-Roch number. Moreover, $H^{0}\left(\Sigma, E_{\lambda}\right) \cong H^{0}\left(\pi, F_{\lambda}\right)=$ $\left\{v \in F_{\lambda} \mid A d \phi\right.$ fixes $\left.v\right\}$ gives rise to the maximal trivial subbundle of $E_{\lambda}$ and a similar description for $E_{-\lambda}$ gives us the equality $\operatorname{dim} H^{0}\left(\Sigma, E_{\lambda}\right)=$ $\operatorname{dim} H^{0}\left(\Sigma, E_{-\lambda}\right)$ via the isomorphism $E_{-\lambda} \cong E_{\lambda}^{*}$. Therefore, we conclude that $\operatorname{dim} H^{0,1}\left(\Sigma, E_{\lambda}\right)=\operatorname{dim} H^{0,1}\left(\Sigma, E_{-\lambda}\right)$ and thus the weights of the $\mathfrak{t}_{\mathfrak{h}}$ action on $W_{\mathfrak{h}}$ are symmetric with respect to the origin. In particular, the action is linearly balanced.

Next, we consider the $\mathfrak{k}$ fixed point subspace $W_{\phi}^{\mathfrak{k}}$ for another infinitesimal stabilizer $\mathfrak{k} \subset \mathfrak{h}$. Let $K$ be the connected subgroup of $S t a b \phi$ whose Lie algebra is $\mathfrak{k}$. Notice that in our case

$$
W_{\phi}^{\mathfrak{k}}=H^{1}\left(\pi,\left(\mathfrak{z}(\mathfrak{h})^{\perp} \cap \mathfrak{z}(\mathfrak{k})\right)_{A d \phi}\right) .
$$

$\mathfrak{z}(\mathfrak{k}) \otimes \mathbb{C}$ is an ideal in the complexification of the Lie algebra of $N^{K}$ and thus the weights of the $N^{K}$ action on $\mathfrak{z}(\mathfrak{k}) \otimes \mathbb{C}$ by conjugation are symmetric with respect to the origin. As $K$ fixes $\mathfrak{z}(\mathfrak{k}) \otimes \mathbb{C}$, the same is true for the action of $N^{K} / K$ on $\mathfrak{z}(\mathfrak{k}) \otimes \mathbb{C}$ and hence so is the action of the subgroup $H \cap N^{K} / K$. Now, $H \cap N^{K} / K$ acts trivially on $\mathfrak{z}(\mathfrak{h}) \otimes \mathbb{C}$ and therefore the weights of the $N^{K} \cap H / K$ action on $\left(\mathfrak{z}(\mathfrak{h})^{\perp} \cap \mathfrak{z}(\mathfrak{k})\right) \otimes \mathbb{C}$ are symmetric with respect to the origin. As in the previous paragraph, we can deduce that the $N^{K} \cap H / K$ 
action on $W_{\phi}^{\mathfrak{k}}$ is also linearly balanced. In summary, we proved the following theorem.

Theorem 4.4 The $G$ action on $U=\mu^{-1}(B) \subset \mathcal{N}^{\mathfrak{g}}$ is balanced. Therefore, we have the isomorphism

$$
I H^{*}(X(G)) \cong V_{U}^{*} \subset H_{G}^{*}(\operatorname{Hom}(\pi, G)) .
$$

Moreover, the above isomorphism preserves the mapping class group action since it is defined by pulling back certain differential forms [Ki] via the quotient map $U \rightarrow X$ which is equivariant with respect to the mapping class group $\Gamma_{g, 1}$ of the punctured Riemann surface.

The equivariant cohomology $H_{G}^{*}(\operatorname{Hom}(\pi, G))$ is isomorphic to the gauge group equivariant cohomology of the space of flat $G$ connections on $\Sigma$, which can be computed by the Morse theory of [AB82]. The cohomology ring $H_{S U(2)}^{*}(\operatorname{Hom}(\pi, S U(2)))$ was completely determined in [Kie00] and hence the above theorem can be applied to compute the intersection cohomology of the representation space as we will see in the next section.

\section{$5 \quad S U(2)$ case}

In this section, we focus on the case where $G=S U(2)$. First, we recall the structure theorem of the equivariant cohomology which determines the cup product structure.

Let $\tilde{\Sigma}$ be the universal cover of $\Sigma$. Consider

$$
\tilde{\Sigma} \times_{\pi}\left(\operatorname{Hom}(\pi, S U(2)) \times \operatorname{End}\left(\mathbb{C}^{2}\right)\right) \rightarrow \Sigma \times \operatorname{Hom}(\pi, S U(2))
$$

where $g \in \pi$ maps $(\phi, v) \in \operatorname{Hom}(\pi, S U(2)) \times \operatorname{End}\left(\mathbb{C}^{2}\right)$ to $(\phi, A d \phi(g) v)$. It is a vector bundle of rank 4 which induces $E n d \mathcal{U}$ over $\Sigma \times\left(\operatorname{Hom}(\pi, S U(2)) \times_{P U(2)}\right.$ $E P U(2))$ by pulling back and taking quotient. We define equivariant classes $\alpha, \beta, \psi_{i}$ of degree $2,4,3$ respectively by

$$
c_{2}(\operatorname{End}(\mathcal{U}))=2 \alpha \otimes[\Sigma]+4 \sum_{i=1}^{2 g} \psi_{i} \otimes e_{i}-\beta \otimes 1
$$

where $e_{i}$ is a symplectic basis of $H^{1}(\Sigma)$ so that $e_{i} e_{i+g}=[\Sigma]$. 
Theorem 5.1 Kie00 Let $\gamma=-2 \sum_{i=1}^{g} \psi_{i} \psi_{i+g}$ and consider the Lefschetz decompositon of the exterior algebra

$$
\wedge\left(\psi_{1}, \cdots, \psi_{2 g}\right)=\bigoplus_{l=1}^{g} \operatorname{Prim}_{l} \otimes \mathbb{C}[\gamma] / \gamma^{g-l+1}
$$

where Prim $_{l}$ is the degree $3 l$ primitive part. Then we have

$$
H_{S U(2)}^{*}(\operatorname{Hom}(\pi, S U(2))) \cong \oplus_{l=0}^{g} \operatorname{Prim}_{l} \otimes \mathbb{C}[\alpha, \beta, \gamma] / I_{g-l}
$$

Here $I_{k}$ is the ideal of the polynomial ring $\mathbb{C}[\alpha, \beta, \gamma]$ generated by $c_{k+1}, c_{k+2}, c_{k+3}$ where the sequence $\left\{c_{n}\right\}$ is defined by

$$
n c_{n}=\alpha c_{n-1}+(n-2) \beta c_{n-2}+2 \gamma c_{n-3}
$$

with $c_{0}=1, c_{1}=\alpha, c_{2}=\frac{\alpha^{2}}{2}$, etc.

Because $\operatorname{Prim}_{l} \mathrm{~s}$ are the irreducible $S p_{2 g}$ modules, this theorem completely describes the mapping class group action as it factors through the symplectic group action on $\psi_{i}$. Furthermore, a Gröbner basis for $I_{k}$ was given in KRie00 so that the cup product can be computed efficiently.

Now by the splitting theorem above, $I H^{*}(X(S U(2)))$ is isomorphic to $V_{U}^{*}$ which we intend to compute. Note that up to conjugation $\left\{S U(2), S^{1}\right\}$ are the only possible stabilizers of points in $\operatorname{Hom}(\pi, S U(2))$. It turns out that we don't have to think about truncation on the $S U(2)$ fixed point set because it is taken care of by that for $S^{1}$. For that one has only to observe that $Y_{S U(2)} \subset Y_{S^{1}}$ and that $n_{S U(2)}=3 g-3>n_{S^{1}}=2 g-3$.

We have to consider the following map

$$
H_{S U(2)}^{*}(\operatorname{Hom}(\pi, S U(2))) \rightarrow H_{S U(2)}^{*}\left(S U(2) \times_{N^{S^{1}}} Y_{S^{1}}\right)=\left[H^{*}(J a c) \otimes \mathbb{C}[u]\right]^{\mathbb{Z} / 2}
$$

where $J a c$ denotes the Jacobian variety and $\mathbb{Z} / 2$ acts as -1 on both components. Let $d_{i}$ be the basis of $H^{1}(J a c)$ defined as the Künneth coefficients of the first Chern class of a universal line bundle corresponding to $e_{i}$. According to CLM00, $\alpha$ is mapped to $w=-2 \sum_{i=1}^{g} d_{i} d_{i+g}, \beta$ to $4 u^{2}$, and $\psi_{i}$ to $-2 u d_{i}$.

Let $P_{t}^{S U(2)}(\operatorname{Hom}(\pi, S U(2)))$ denote the Poicaré series for the equivariant cohomology $H_{S U(2)}^{*}(\operatorname{Hom}(\pi, S U(2)))$. Then it is well known from AB82 that

$$
P_{t}^{S U(2)}(\operatorname{Hom}(\pi, S U(2)))=\frac{\left(1+t^{3}\right)^{2 g}-t^{2 g+2}(1+t)^{2 g}}{\left(1-t^{2}\right)\left(1-t^{4}\right)} .
$$


For the intersection Poicaré series $I P_{t}(X)=\sum t^{i} \operatorname{dim} I H^{i}(X)$, we have only to subtract out the Poincare series of

$$
\operatorname{Im}\left\{H_{S U(2)}^{*}(\operatorname{Hom}(\pi, S U(2))) \rightarrow\left[H^{*}(J a c) \otimes \mathbb{C}[u]\right]^{\mathbb{Z} / 2}\right\} \cap\left\{H^{*}(J a c) \otimes u^{g-1} \mathbb{C}[u]\right\} .
$$

By the Lefshetz decomposition and a trivial combinatorial argument, one can easily see that the intersection is actually

$$
\left[H^{*}(J a c) \otimes u^{g-1} \mathbb{C}[u]\right]^{\mathbb{Z} / 2}
$$

whose Poincare series is precisely

$$
\frac{1}{2}\left\{\frac{(1+t)^{2 g}\left(t^{2}\right)^{g-1}}{1-t^{2}}+\frac{(1-t)^{2 g}\left(-t^{2}\right)^{g-1}}{1+t^{2}}\right\} .
$$

\section{Proposition 5.2}

$I P_{t}(X(S U(2)))=P_{t}^{S U(2)}(\operatorname{Hom}(\pi, S U(2)))-\frac{1}{2}\left\{\frac{(1+t)^{2 g}\left(t^{2}\right)^{g-1}}{1-t^{2}}+\frac{(1-t)^{2 g}\left(-t^{2}\right)^{g-1}}{1+t^{2}}\right\}$

It is an elementary exercise to check that Proposition 5.2 indeed coincides with Kirwan's computations [Kir86].

A little bit more careful examination of the truncation map together with Theorem 5.1 described above gives us the following.

Theorem 5.3 Let $E_{m}$ be the vector space spanned by

$$
\left\{\alpha^{i} \beta^{j} \xi^{k} \mid(1) i+2 k \leq m, \text { (2) } j+2 k \leq m \text {, (3) if } k=0 \text { then } j<\left[\frac{m}{2}\right]\right\}
$$

where $\xi=\alpha \beta+2 \gamma$. Then

$$
I H^{*}(X(S U(2))) \cong \oplus_{i=0}^{g} \operatorname{Prim}_{l} \otimes E_{g-l} .
$$

Again, this theorem precisely describes the action of the mapping class group $\Gamma_{g, 1}$ as our isomorphism is equivariant and hence the action also factors through the symplectic group action on Prim . $_{\text {. }}$.

Also the intersection pairing can be computed in terms of the cup product structure. One can prove that

$$
\alpha^{m} \beta^{n}=-m ! b_{g-n-1} \frac{\alpha^{g-2} \beta^{g-2} \xi}{(g-2) !}
$$


where $m+2 n=3 g-3, n<g-1$ and $b_{k}$ are given by

$$
\frac{t}{\tanh t}=\sum_{k \geq 0} b_{k} t^{2 k}
$$

directly from the structure theorem at least for low genus case. Hence, if we take $\frac{\alpha^{g-2} \beta^{g-2} \xi}{(g-2) !(-4)^{g-1}}$, as our fundamental class then we get

$$
\left\langle\kappa\left(\alpha^{i} \beta^{j}\right), \kappa\left(\alpha^{k} \beta^{l}\right)\right\rangle=-(-4)^{g-1} m ! b_{g-n-1}
$$

for $i+k=m, j+l=n, m+2 n=3 g-3, n<g-1$. In principle, it is a number theoretic or combinatorial exercise to deduce the above formula from the structure theorem but it seems very difficult to achieve in practice.

However the above pairing formula can be justified by using the computation of intersection numbers on the moduli spaces of parabolic bundles by Jeffrey and Kirwan [JK98. This will be done in [JKKW].

\section{References}

[AB82] M.F. Atiyah and R. Bott. The Yang-Mills equations over Riemann surfaces. Phil. Trans. Roy. Soc. Lond., A308:532-615, 1982.

[CLM00] S. Cappell, R. Lee, and E. Miller. The action of the torelli group on the homology of representation spaces is nontrivial. Topology, 39(4):851-871, 2000.

[GM88] W. Goldman and J. Millson. The deformation theory of representations of fundamental groups of compact kähler manifolds. Publ. IHES, 67:43-96, 1988.

[Gol84] W. Goldman. The symplectic nature of fundamental groups of surfaces. Adv. Math., 54:200-225, 1984.

[Jef94] L.C. Jeffrey. Extended moduli spaces of flat connections on Riemann surfaces. Math. Annalen, 298:667-692, 1994.

[JK98] L. Jeffrey and F. Kirwan. Intersection theory on moduli spaces of holomorphic bundles of arbitrary rank on a Riemann surface. Ann. Math., 148:109-196, 1998. 
[JKKW] L. Jeffrey, Y.-H. Kiem, F. Kirwan, and J. Woolf. Intersection pairings on singular moduli spaces of bundles over a Riemann surface. In preparation.

[Kie] Y.-H. Kiem. Intersection cohomology of quotients of nonsingular varieties. Preprint.

[Kie00] Y.-H. Kiem. The equivariant cohomology ring of the moduli space of vector bundles over a riemann surface. Contemporary Mathematics, 258:249-261, 2000.

[Kir84] F. Kirwan. Cohomology of Quotients in Symplectic and Algebraic Geometry. Number 34 in Mathematical Notes. Princeton University Press, 1984.

[Kir86] F. Kirwan. On the homology of compactifications of moduli spaces of vector bundles over a riemann surface. Proc. Lon. Math. Soc., 53:237-266, 1986.

[KW] Y.-H. Kiem and J. Woolf. The cosupport axiom, equivariant cohomology and the intersection cohomology of certain symplectic quotients. Preprint.

[Ram75] A. Ramanathan. Stable principal bundles on a compact riemann surface. Math. Ann., 213:129-152, 1975.

[SL91] R. Sjamaar and E. Lerman. Stratified symplectic spaces and reduction. Annals of Maths, 134:375-422, 1991. 